\title{
Four-node shell element for doubly curved multilayered composites based on the Refined Zigzag Theory
}

\author{
Daniele Versino*, Marco Gherlone ${ }^{b}$, Marco Di Sciuva $^{b}$ \\ *Theoretical Division, T-3, Los Alamos National Laboratory, MS B216, Los Alamos, New Mexico 87545, USA \\ ${ }^{b}$ Department of Mechanical and Aerospace Engineering, Politecnico di Torino, Corso Duca degli Abruzzi 24, 10129 Torino, Italy
}

\begin{abstract}
In the present paper a generalization of the Refined Zigzag Theory (RZT) to doubly-curved multilayered structures is proposed. The displacement field characteristic of Naghdi's shell model is enriched with RZT kinematics and a four-node shell finite element is formulated. Assumed Natural Strain (ANS) strategy is employed to overcome shear locking and Enhanced Assumed Strain (EAS) technique is applied to alleviate membrane locking and bending locking. For efficiency purpose, a one-point quadrature rule is used for the in-plane integration and hourglass stabilization is introduced. Finally, several numerical examples, involving static analysis of thick as well as thin shells, are performed to demonstrate the efficiency and accuracy of the proposed shell finite element.
\end{abstract}

Keywords: Laminated composites, Refined Zigzag Theory, doubly curved shell element, reduced integration, small deformations.

\section{Introduction}

In the last decades there has been a recognizable increase in the application of composite materials within a lot of engineering fields (aerospace and marine, civil and defense). This success is mainly due to the fact that composite materials are stiff and resistant light-weight materials and that they can be easily tailored in order to be optimized for any specific use. The typical structural typology built up with composite materials is based on a thickness-wise sequence of layers with different mechanical properties (transverse anisotropy). Taking into account that composite materials also exhibit a transverse deformability that is higher than for metallic materials, the modeling effort is particularly challenging.

The usual classifications adopted for the one- and twodimensional models of multilayered composite structures are based on the nature of the primary unknowns and on the assumptions made on these unknowns. There are displacementbased theories (only the displacements are primary unknowns) [1] and mixed theories (both the displacements and the transverse stresses may be unknowns) [2]. The focus of the present work will be on displacement-based theories. Moreover, depending on the through-the-thickness description of the displacement and stress fields, there may be: Equivalent Single Layer theories (ESL, a through-the-thickness distribution of the unknowns is assumed over the whole laminate thickness) and Layer-Wise theories (LW, the distribution of the unknowns is assumed layer by layer) [3].

The Classical Lamination Plate Theory (CLPT) [4] and the First-order Shear Deformation Theory (FSDT) [5, 6] are easy-

\footnotetext{
${ }^{*}$ Corresponding author. Tel: +1 505667 2137. Fax: +1 5056655926.
}

Email address: daniele.versino@lanl.gov (Daniele Versino) to-implement displacement-based ESL theories widely adopted for research and engineering applications. Moreover, most of the finite elements present into commercial codes are based on the FSDT. Nevertheless, both CLPT and FSDT do not model accurately transverse strains and stresses: CLPT totally neglects transverse deformability whereas FSDT produces piecewise constant transverse shear stresses, thus violating the requirement of transverse stresses through-the-thickness continuity. For these reasons, CLPT and FSDT may be used for thin and moderately thick laminated structures with reduced transverse anisotropy and their applicability is often limited to global responses (maximum deflection, vibration frequencies, buckling loads). Higher-order through-the-thickness expansions of the displacements (polynomial [7, 8] or trigonometric [9]) may lead to improved predictions but transverse stresses continuity is not satisfied (unless integration of indefinite equilibrium equations is applied to recover accurate transverse stresses).

LW theories based on displacements $[10,11]$ can guarantee a high degree of accuracy, but at the expense of computational complexity. The number of unknowns in LW theories increases with the number of layers thus becoming prohibitive for complex analyses (non-linear, progressive failure) on laminated structures with several layers.

An interesting compromise between high accuracy and low computational costs is represented by the so-called zigzag theories. These theories were pioneered in the early eighties by Di Sciuva [12-14]: transverse normal deformability is usually neglected and transverse displacement is through-the-thickness constant whereas in-plane displacements are the result of the superposition of a through-the-thickness polynomial distribution (linear [13] or cubic [14]) and a piecewise linear and continuous (i.e., zigzag) contribution, introduced in order to model the thickness-normal distortion typical of multilayered struc- 
tures. The zigzag contribution to the in-plane displacement is shaped in order to guarantee that transverse shear stresses are through-the-thickness continuous. The total number of unknowns does not depend on the number of layers. Inspired by Di Sciuva's works, a great number of zigzag theories have been proposed over the last two decades [15-23].

Recently, a further development has been proposed within the family of zigzag models, namely the Refined Zigzag Theory (RZT) [24-27], in order to consistently address some drawbacks of the previously presented approaches. In fact, the transverse shear stresses obtained from the constitutive equations of classical zigzag theories vanish erroneously at clamped boundaries and this has been ignored by many authors (apart from Averill [16, 17]). Within RZT, transverse shear stresses are allowed to be piecewise constant and discontinuous at the layer interfaces. Nevertheless, more accurate predictions of all response quantities are obtained, including the transverse shear stresses, also at clamped boundaries [24, 26, 28]. Moreover, most zigzag theories require $\mathrm{C} 1$-continuous finite element approximations, the type of approximation that is particularly undesirable for plate and shell elements. Some of the already mentioned works have faced this problem [16, 17, 23]. A number of finite element formulations based on RZT have been presented [29-34], all of them requiring only C0-continuity of shape functions and thus being suitable for inclusion into FEM commercial codes [31]. Finally, a great versatility of RZT has been demonstrated, since the zigzag contribution to the in-plane displacements can be naturally and accurately adapted to some special cases, including homogeneous laminates [27] and laminates with external shear-deformable layers [35]. Further enhancements of RZT can be found in [36, 37].

In the present paper a generalization of RZT to doublycurved multilayered shells is introduced. A four-node shell element based on the proposed formulation is developed employing bilinear shape functions for the in-plane interpolation of the seven kinematic variables. Therefore, seven degrees of freedom are defined at each node of the element, namely three displacements, two average rotations and two zig-zag amplitudes. The current formulation may be regarded as an extension of previous work by Versino et al. [31]. Shear locking due to the bilinear interpolation is mitigated using the assumed natural strain (ANS) interpolation [32, 38, 39]. Moreover, the element's performances in membrane- and bending-dominated problems are improved applying the enhanced assumed strain (EAS) methodology [40, 41]. In-plane integration is carried out with a one-point quadrature rule and hourglass stabilization is employed [42, 43].

The paper is organized as follows. In the next section, the problem is described and the notation is defined. In Section 3, geometric and kinematic quantities of the shell are introduced through a tensorial representation. The displacement field of the RZT-based shell is presented in Section 4. Subsequently, the variational formulation of the equilibrium equations is derived in Section 5, and the resulting computational formulation is derived in Section 6. The performances of the present formulation are assessed by way of several numerical experiments, involving thick as well as thin shells, in Section 7. Finally, con- cluding remarks are presented in Section 8.

\section{Notation}

A curved laminated shell of thickness $2 h$ is composed of $N$ perfectly-bonded layers. The $N$ layers are separated by $N-1$ interfaces and the notation $(\bullet)^{(k)}$ is used to indicate a quantity associated with the $k$-th layer or interface. The Cartesian coordinates of every point belonging to the shell's reference surface, $\Omega$, are given by its position vector $\boldsymbol{r}\left(\xi^{1}, \xi^{2}\right)$, where $\xi^{1}, \xi^{2}$ are curvilinear coordinates defined on the closed domain $\Omega$. The $\xi^{3}$-coordinate spans the range $[-h, h]$, and is directed along the unit vector $\boldsymbol{a}_{3}\left(\xi^{1}, \xi^{2}\right)$, normal to the reference surface (see

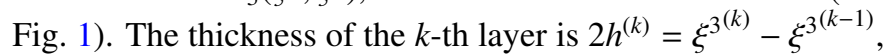
the $k$-th interface is situated between layers $k$ and $k+1$ and the top and bottom surfaces of the shell are respectively located at $\xi^{3^{(N)}}=h$ and $\xi^{3^{(0)}}=-h$. The Cartesian coordinates of every point in the closed domain occupied by the shell, $\mathscr{B}=\Omega \times[-h, h]$, are hence given by its position vector,

$$
\boldsymbol{R}\left(\xi^{1}, \xi^{2}, \xi^{3}\right)=\boldsymbol{r}\left(\xi^{1}, \xi^{2}\right)+\xi^{3} \boldsymbol{a}_{3} .
$$

The domain's boundary, $\partial \mathscr{B}$, is split into two disjoint subsets, $\partial \mathscr{B}_{u}=\partial \Omega_{u} \times[-h, h]$ and $\partial \mathscr{B}_{\sigma}=\partial \Omega_{\sigma} \times[-h, h]$, where essential, $\boldsymbol{u}=\overline{\boldsymbol{u}}$, and natural boundary conditions, $\boldsymbol{\sigma} \cdot \boldsymbol{a}_{3}=\overline{\boldsymbol{h}}$, respectively, are enforced. Finally, it is noted that in the following, unless otherwise stated, Greek indices vary from 1 to 2 , while their Latin counterparts vary from 1 to 3 .

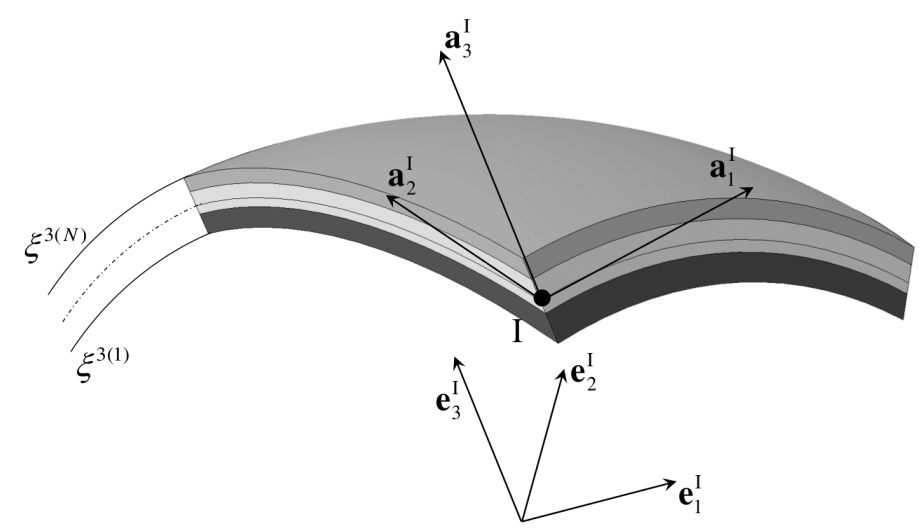

Figure 1: Shell geometry.

\section{Kinematic description}

It is recalled from the previous section that the midsurface of the shell is defined by the mapping, $\boldsymbol{r}$, of convected curvilinear coordinates, $\xi^{\alpha}$, into Cartesian coordinates in $\mathbb{R}^{3}$. The covariant base vectors of the tangential plane are thus given by [44]:

$$
\boldsymbol{a}_{\alpha}=\frac{\partial \boldsymbol{r}}{\partial \xi^{\alpha}} .
$$

The components of the surface metric tensor (first fundamental form) are defined as

$$
a_{\alpha \beta}=\boldsymbol{a}_{\alpha} \cdot \boldsymbol{a}_{\beta},
$$


and the following properties hold:

$$
a^{\alpha \beta}=\left(a_{\alpha \beta}\right)^{-1}, \quad \boldsymbol{a}^{\alpha}=a^{\alpha \beta} \boldsymbol{a}_{\beta}, \quad \boldsymbol{a}_{\alpha} \cdot \boldsymbol{a}^{\beta}=\delta_{\alpha}^{\beta},
$$

where $\delta_{\alpha}^{\beta}$ is Kronecker's delta tensor. Moreover, the unit normal vector is given by

$$
a_{3}=\frac{a_{1} \times a_{2}}{\left\|a_{1} \times a_{2}\right\|}
$$

and an element of surface area is obtained as

$$
\mathrm{d} \Omega=\left\|\boldsymbol{a}_{1} \times \boldsymbol{a}_{2}\right\| \mathrm{d} \xi^{1} \mathrm{~d} \xi^{2}=\sqrt{\operatorname{det}\left(a_{\alpha \beta}\right)} \mathrm{d} \xi^{1} \mathrm{~d} \xi^{2} .
$$

In light of the mapping (1), three-dimensional covariant base vectors for the $\boldsymbol{\xi}$-reference frame are given by

$$
\boldsymbol{g}_{i}=\frac{\partial \boldsymbol{R}}{\partial \xi^{i}}
$$

the components of the metric tensor are given in covariant form as

$$
g_{i j}=g_{i} \cdot g_{j}
$$

and, similarly to Eqs. (4), the following properties hold:

$$
g^{i j}=\left(g_{i j}\right)^{-1}, \quad \boldsymbol{g}^{i}=g^{i j} \boldsymbol{g}_{j}, \quad \boldsymbol{g}_{i} \cdot \boldsymbol{g}^{j}=\delta_{i}^{j}
$$

Substituting Eq. (1) into (7), the covariant base vectors $\boldsymbol{g}_{i}$ may be expressed as follows

$$
\boldsymbol{g}_{\alpha}=\boldsymbol{a}_{\alpha}+\xi^{3} \boldsymbol{a}_{3, \alpha}, \quad \quad \boldsymbol{g}_{3}=\boldsymbol{a}_{3}
$$

Following Ref. [39], $\boldsymbol{a}_{3}$ is considered here and henceforth to be an approximation to the reference surface normal given by Eq. (5). Accordingly, it is not treated as strictly orthogonal to $\boldsymbol{a}_{\alpha}$ in the above expressions.

Quantities computed in the reference frame defined by the covariant base $\boldsymbol{g}_{i}$ are mapped to the reference surface using the shifter tensor, given by [45]:

$$
\mu_{\beta}^{\alpha}=\boldsymbol{a}_{\beta} \cdot \boldsymbol{g}^{\alpha}
$$

Thus, an element of area at a given $\xi^{3}=z$ is given by

$$
\left.\mathrm{d} \Omega\right|_{\xi^{3}=z}=\operatorname{det}\left(\left.\mu_{\beta}^{\alpha}\right|_{\xi^{3}=z}\right),
$$

where

$$
\mu^{(k)}=\left.\mu\right|_{\xi^{3(k)}},
$$

and

$$
\mu=\operatorname{det}\left(\mu_{\beta}^{\alpha}\right)
$$

The mapping between corresponding points in the initial and in the current (deformed) configurations is indicated with $\hat{\boldsymbol{R}}$

$$
\hat{\boldsymbol{R}}\left(\xi^{i}\right)=\boldsymbol{R}\left(\xi^{i}\right)+\boldsymbol{u}\left(\xi^{i}\right)
$$

where $(\hat{\bullet})$ denotes a quantity in the deformed configuration. The components of the Green-Lagrange strain tensor are defined as follows [46]:

$$
\varepsilon_{i j}=\frac{1}{2}\left(\hat{g}_{i j}-g_{i j}\right)=\frac{1}{2}\left(\frac{\partial \hat{\boldsymbol{R}}}{\partial \xi^{i}} \cdot \frac{\partial \hat{\boldsymbol{R}}}{\partial \xi^{j}}-\frac{\partial \boldsymbol{R}}{\partial \xi^{i}} \cdot \frac{\partial \boldsymbol{R}}{\partial \xi^{j}}\right) .
$$

where $\hat{g}_{i j}$ denotes the metric tensor in the current configuration. Combining (15) and (16) leads to

$$
\varepsilon_{i j}=\frac{1}{2}\left(\frac{\partial(\boldsymbol{R}+\boldsymbol{u})}{\partial \xi^{i}} \cdot \frac{\partial(\boldsymbol{R}+\boldsymbol{u})}{\partial \xi^{j}}-\frac{\partial \boldsymbol{R}}{\partial \xi^{i}} \cdot \frac{\partial \boldsymbol{R}}{\partial \xi^{j}}\right),
$$

and using Eq. (7), we obtain

$$
\varepsilon_{i j}=\frac{1}{2}\left(\boldsymbol{g}_{i} \cdot \frac{\partial \boldsymbol{u}}{\partial \xi^{j}}+\frac{\partial \boldsymbol{u}}{\partial \xi^{i}} \cdot \boldsymbol{g}_{j}+\frac{\partial \boldsymbol{u}}{\partial \xi^{i}} \cdot \frac{\partial \boldsymbol{u}}{\partial \xi^{j}}\right) .
$$

Assuming small strains, we neglect the non-linear term in the above expression, and write

$$
\varepsilon_{i j}=\frac{1}{2}\left(\boldsymbol{g}_{i} \cdot \frac{\partial \boldsymbol{u}}{\partial \xi^{j}}+\frac{\partial \boldsymbol{u}}{\partial \xi^{i}} \cdot \boldsymbol{g}_{j}\right)
$$

\section{RZT displacement field and strain tensor}

At a given point of the shell it is possible to define a Cartesian reference frame, defined by the basis $\left[\boldsymbol{V}_{1}, \boldsymbol{V}_{2}, \boldsymbol{V}_{3}\right]$, with

$$
V_{3} \equiv a_{3}, \quad V_{1}=\frac{a_{2} \times a_{3}}{\left\|a_{2} \times a_{3}\right\|}, \quad V_{2}=V_{3} \times V_{1}
$$

The displacement field of a shell with Refined Zigzag kinematics is given, with respect to the global Cartesian reference frame, as

$$
\boldsymbol{u}^{(k)}=\boldsymbol{u}_{0}+\xi^{3}\left(-\boldsymbol{V}_{2} \theta_{1}+\boldsymbol{V}_{1} \theta_{2}\right)-\phi_{1}^{(k)} \boldsymbol{V}_{2} \psi_{1}+\phi_{2}^{(k)} \boldsymbol{V}_{1} \psi_{2}
$$

where $\boldsymbol{u}_{0}=\left\{u_{1}, u_{2}, u_{3}\right\}$ represents the mid-surface displacement vector, $\theta_{1}$ and $\theta_{2}$ denote the average rotations around the $\boldsymbol{V}_{2}$ and $\boldsymbol{V}_{1}$ axis respectively, $\phi_{1}^{(k)}$ and $\phi_{2}^{(k)}$ denote the zigzag functions of the $k$-th layer and $\psi_{1}$ and $\psi_{2}$ are respectively the zigzag rotation around the $\boldsymbol{V}_{2}$ axis and the zigzag rotation around the $\boldsymbol{V}_{1}$ axis. Introducing the following quantities

$$
\begin{array}{ll}
\boldsymbol{\theta}=-\boldsymbol{V}_{2} \theta_{1}+\boldsymbol{V}_{1} \theta_{2}, & \\
\boldsymbol{\psi}_{1}=-\boldsymbol{V}_{2} \psi_{1}, & \boldsymbol{\psi}_{2}=\boldsymbol{V}_{1} \psi_{2},
\end{array}
$$

allows the displacement field of the $k$-th layer to be rewritten as

$$
\boldsymbol{u}^{(k)}=\boldsymbol{u}_{0}+\xi^{3} \boldsymbol{\theta}+\phi_{1}^{(k)} \boldsymbol{\psi}_{1}+\phi_{2}^{(k)} \boldsymbol{\psi}_{2}
$$

The Green-Lagrange strain of the $k$-th layer is hence obtained by substituting Eq. (24) into Eq. (19) and can be written in terms of strain measures as

$$
\begin{aligned}
\boldsymbol{\varepsilon}^{(k)}=\boldsymbol{\varepsilon}^{\mathrm{m}}+\boldsymbol{\varepsilon}^{\mathrm{s}}+\xi^{3} \boldsymbol{\varepsilon}^{\mathrm{b}} & +\phi_{1}^{(k)} \boldsymbol{\varepsilon}^{\mathrm{b}_{\phi_{1}}}+\phi_{2}^{(k)} \boldsymbol{\varepsilon}^{\mathrm{b}_{\phi_{2}}} \\
& +\beta_{1}^{(k)} \boldsymbol{\varepsilon}^{\mathrm{s}_{\phi_{1}}}+\beta_{2}^{(k)} \boldsymbol{\varepsilon}^{\mathrm{s}_{\phi_{2}}}
\end{aligned}
$$


with $\beta_{\alpha}^{(k)}=\partial \phi_{\alpha}^{(k)} / \partial \xi^{3}$ for $\alpha=1,2$, where the membrane, bending and shear strain measures denoted respectively with $\boldsymbol{\varepsilon}^{\mathrm{m}}, \boldsymbol{\varepsilon}^{\mathrm{b}}$ and $\varepsilon^{\mathrm{s}}$ are given by

$$
\begin{aligned}
& \varepsilon_{\alpha \beta}^{\mathrm{m}}=\frac{1}{2}\left(\boldsymbol{u}_{0, \alpha} \cdot \boldsymbol{a}_{\beta}+\boldsymbol{u}_{0, \beta} \cdot \boldsymbol{a}_{\alpha}\right), \\
& \varepsilon_{\alpha \beta}^{\mathrm{b}}=\frac{1}{2}\left(\boldsymbol{u}_{0, \alpha} \cdot \boldsymbol{a}_{3, \beta}+\boldsymbol{u}_{0, \beta} \cdot \boldsymbol{a}_{3, \alpha}+\boldsymbol{\theta}_{, \alpha} \cdot \boldsymbol{a}_{\beta}+\boldsymbol{\theta}_{, \beta} \cdot \boldsymbol{a}_{\alpha}\right), \\
& \varepsilon_{\alpha 3}^{\mathrm{s}}=\frac{1}{2}\left(\boldsymbol{u}_{0, \alpha} \cdot \boldsymbol{a}_{3}+\boldsymbol{\theta} \cdot \boldsymbol{a}_{\alpha}\right) .
\end{aligned}
$$

and where the following assumptions on the strain tensor have been made: 1) in-plane strains are at most linear in the throughthickness coordinate, 2) transverse shear strains are constant along the through-thickness coordinate. Moreover, the zigzag bending strain measures, $\varepsilon_{\alpha \beta}^{\mathrm{b}_{\phi_{1}}}, \varepsilon_{\alpha \beta}^{\mathrm{b}_{\phi_{2}}}$ and $\varepsilon_{\alpha \beta}^{\mathrm{b}_{\phi_{3}}}$, and the zigzag transverse shear strain measures, $\varepsilon_{\alpha 3}^{\mathrm{s}_{\phi_{1}}}, \varepsilon_{\alpha 3}^{\mathrm{s}_{\phi_{2}}}$ and $\varepsilon_{\alpha 3}^{\mathrm{s}_{\phi_{3}}}$, are obtained from

$$
\begin{aligned}
\varepsilon_{\alpha \beta}^{\mathrm{b}_{\phi_{1}}} & =\frac{1}{2}\left(\psi_{1, \alpha} \cdot \boldsymbol{a}_{\beta}+\psi_{1, \beta} \cdot \boldsymbol{a}_{\alpha}\right), \\
\varepsilon_{\alpha \beta}^{\mathrm{b}_{\phi_{2}}} & =\frac{1}{2}\left(\psi_{2, \alpha} \cdot \boldsymbol{a}_{\beta}+\psi_{2, \beta} \cdot \boldsymbol{a}_{\alpha}\right), \\
\varepsilon_{\alpha 3}^{\mathrm{s}_{\phi_{1}}} & =\frac{1}{2} \psi_{1} \cdot \boldsymbol{a}_{\alpha}, \\
\varepsilon_{\alpha 3}^{\mathrm{s}_{\phi_{2}}} & =\frac{1}{2} \psi_{2} \cdot \boldsymbol{a}_{\alpha} .
\end{aligned}
$$

It should be remarked that the transverse normal strain, $\varepsilon_{33}$, is identically zero and that the zigzag transverse shear strains are piecewise constant along the $\xi^{3}$ coordinate.

\section{Variational formulation}

The variational form of the principle of conservation of linear momentum, when body forces are neglected and under the assumption of homogeneous Dirichlet boundary conditions, can be written as follows (Refs. [40])

$$
\begin{aligned}
\int_{\Omega} \int_{h} \delta \boldsymbol{\varepsilon}: \boldsymbol{\sigma} \mu \mathrm{d} \xi^{3} \mathrm{~d} \Omega & =\int_{\partial \Omega_{\sigma}} \int_{h} \delta \boldsymbol{u} \cdot \overline{\boldsymbol{h}} \mu \mathrm{d} \xi^{3} \mathrm{~d} \Gamma \\
& +\left.\int_{\Omega} \delta \boldsymbol{u}\right|_{\xi^{3^{(0)}}} \cdot \boldsymbol{a}_{3} q^{(0)} \mu^{(0)} \mathrm{d} \Omega \\
& -\left.\int_{\Omega} \delta \boldsymbol{u}\right|_{\xi^{3^{(N)}}} \cdot \boldsymbol{a}_{3} q^{(N)} \mu^{(N)} \mathrm{d} \Omega
\end{aligned}
$$

where $q^{(0)}$ and $q^{(N)}$ respectively identify the distributed loads acting on the bottom and top surfaces of the laminate and where through the thickness integration is indicated by the shorthand notation $\int_{h}(\bullet) \mathrm{d} \xi^{3}$

$$
\int_{h}(\bullet) \mathrm{d} \xi^{3}=\sum_{k=1}^{N} \int_{h^{(k)}}(\bullet) \mathrm{d} \xi^{3}=\sum_{k=1}^{N} \int_{\xi^{3(k-1)}}^{\xi^{3(k)}}(\bullet) \mathrm{d} \xi^{3}
$$

The variational statement in Eq. (33) considers only displacement as primary unknowns. Nevertheless, it is possible to apply the EAS methodology [47] to improve the efficiency of the numerical formulation. Therefore, strain unknowns are introduced and the Green-Lagrange strain is enriched with a strain field discontinuous at the element boundaries

$$
\boldsymbol{\varepsilon}=\overline{\boldsymbol{\varepsilon}}+\tilde{\boldsymbol{\varepsilon}}, \quad \delta \boldsymbol{\varepsilon}=\delta \bar{\varepsilon}+\delta \tilde{\varepsilon},
$$

where $\overline{\boldsymbol{\varepsilon}}$ is the compatible strain field obtained from Eq. (25), and where $\tilde{\boldsymbol{\varepsilon}}$ and $\delta \tilde{\boldsymbol{\varepsilon}}$ denote respectively the unknown enrichments and their virtual variation.

A tessellation $\Omega^{h}$ of the reference surface $\Omega$ is given such as $\Omega^{h}=\bigcup_{e \in \eta_{e}} \Omega^{e}$ where $\Omega^{e}$ denotes the domain of an element with boundaries $\partial \Omega^{e}$ and $\eta_{e}$ is the set of element numbers. The boundary of the reference surface, $\partial \Omega$, may be divided into two subsets such that the closure of $\overline{\partial \Omega}=\overline{\partial \Omega_{u} \cup \partial \Omega_{\sigma}}$ and that $\partial \Omega_{u} \cap \partial \Omega_{\sigma}=\emptyset$ where $\partial \Omega_{u}$ and $\partial \Omega_{\sigma}$ indicate the sets where respectively Dirichlet and Neumann boundary conditions are enforced. Introducing the symbols $\Pi_{\mathrm{I}}^{e}$ and $\Pi_{\mathrm{E}}^{e}$ to denote respectively the element level internal deformation energy and the external work made by the forces applied to the Neumann boundary, the mixed variational statement with displacement and strain enrichments as primary unknowns is given by

$$
\sum_{e \in \eta_{e}}\left(\delta \Pi_{\mathrm{I}}^{e}-\delta \Pi_{\mathrm{E}}^{e}\right)=0
$$

where the internal and external energy contributions are obtained from

$$
\begin{aligned}
\delta \Pi_{\mathrm{I}}^{e} & =\int_{\Omega^{e}} \int_{h}[\delta \overline{\boldsymbol{\varepsilon}}+\delta \tilde{\boldsymbol{\varepsilon}}]: \boldsymbol{\sigma} \mu \mathrm{d} \xi^{3} \mathrm{~d} \Omega^{e}, \\
\delta \Pi_{\mathrm{E}}^{e} & =\int_{\Omega_{\sigma}^{e}} \int_{h} \delta \boldsymbol{u} \cdot \overline{\boldsymbol{h}} \mu \mathrm{d} \xi^{3} \mathrm{~d} \Gamma^{e} \\
& +\int_{\Omega^{e}}\left(\left.\delta \boldsymbol{u}\right|_{\xi^{3(0)}} q^{(0)} \mu^{(0)}-\left.\delta \boldsymbol{u}\right|_{\xi^{3(N)}} q^{(N)} \mu^{(N)}\right) \cdot \boldsymbol{a}_{3} \mathrm{~d} \Omega^{e} .
\end{aligned}
$$

It should be observed that the variational formulation in Eq. (36) is modified in order to include the orthogonality condition, characteristic of EAS method, of the stress field and the strain enrichment, $\tilde{\varepsilon}$,

$$
\int_{\Omega^{e}} \int_{h} \delta \tilde{\varepsilon}: \sigma \mu \mathrm{d} \xi^{3} \mathrm{~d} \Omega^{e}=0 .
$$

Under the assumption of linear elastic orthotropic material behavior, Hooke's law, the stress for the $k$-th layer is given by

$$
\boldsymbol{\sigma}^{(k)}=\mathbb{C}^{(k)}: \boldsymbol{\varepsilon}^{(k)}
$$

where the tangent moduli characteristic of the material of the $k$-th layer, $\mathbb{C}^{(k)}$, is a rank four tensor. Membrane, bending and transverse shear stress section resultants are derived from the through-thickness integration of the stresses. Therefore, the membrane stress resultants are

$$
\mathrm{N}^{\alpha \beta}=\int_{h} \sigma^{\alpha \beta} \mu \mathrm{d} \xi^{3},
$$

the bending stress resultants are

$$
\left\langle\mathbf{M}^{\alpha \beta}, \mathbf{M}_{\phi_{1}}^{\alpha \beta}, \mathbf{M}_{\phi_{2}}^{\alpha \beta}\right\rangle=\int_{h}\left\langle\xi^{3}, \phi_{1}^{(k)}, \phi_{2}^{(k)}\right\rangle \sigma^{\alpha \beta} \mu \mathrm{d} \xi^{3},
$$


and the transverse shear stress resultants are

$$
\left\langle\mathrm{Q}^{\alpha 3}, \mathrm{Q}_{\phi_{1}}^{\alpha 3}, \mathrm{Q}_{\phi_{2}}^{\alpha 3}\right\rangle=\int_{h}\left\langle 1, \beta_{1}^{(k)}, \beta_{2}^{(k)}\right\rangle \sigma^{\alpha 3} \mu \mathrm{d} \xi^{3}
$$

where, due to the symmetry of $\mathbb{C}$, the following equivalencies $\mathrm{Q}^{3 \alpha} \equiv \mathrm{Q}^{\alpha 3}, \mathrm{Q}_{\phi_{1}}^{3 \alpha} \equiv \mathrm{Q}_{\phi_{1}}^{\alpha 3}$ and $\mathrm{Q}_{\phi_{2}}^{3 \alpha} \equiv \mathrm{Q}_{\phi_{2}}^{\alpha 3}$ hold. Zigzag bending moments, $\mathbf{M}_{\phi_{1}}$ and $\mathbf{M}_{\phi_{2}}$, and zigzag transverse shear forces, $\mathbf{Q}_{\phi_{1}}$ and $\mathbf{Q}_{\phi_{2}}$, may be regarded as higher-order stress resultants [31] associated with the zigzag term of the in-plane displacements, see Eq. (24). The virtual variation of the internal energy, Eq. (37), is hence rewritten in terms of the section stress resultants introduced in Eqs. (41)-(43)

$$
\begin{aligned}
& \delta \Pi_{\mathrm{I}}^{e}=\int_{\Omega^{e}}\left[\left(\delta \boldsymbol{\varepsilon}^{\mathrm{m}}+\delta \tilde{\boldsymbol{\varepsilon}}^{\mathrm{m}}\right): \mathbf{N}+\left(\delta \boldsymbol{\varepsilon}^{\mathrm{b}}+\delta \tilde{\boldsymbol{\varepsilon}}^{\mathrm{b}}\right): \mathbf{M}\right. \\
& +\delta \boldsymbol{\varepsilon}^{\mathrm{b}_{\phi_{1}}}: \mathbf{M}_{\phi_{1}}+\delta \boldsymbol{\varepsilon}^{\mathrm{b}_{\phi_{2}}}: \mathbf{M}_{\phi_{2}}+\delta \boldsymbol{\varepsilon}^{\mathrm{s}}: \mathbf{Q} \\
& \left.+\delta \boldsymbol{\varepsilon}^{\mathrm{s}_{\phi_{1}}}: \mathbf{Q}_{\phi_{1}}+\delta \boldsymbol{\varepsilon}^{\mathrm{s}_{\phi_{2}}}: \mathbf{Q}_{\phi_{2}}\right] \mathrm{d} \Omega^{e},
\end{aligned}
$$

where $\tilde{\boldsymbol{\varepsilon}}^{\mathrm{m}}$ and $\tilde{\boldsymbol{\varepsilon}}^{\mathrm{b}}$ denote the enrichment of the membrane and bending strains respectively. Finally, the variation of the external work, Eq. (38), is given by

$$
\begin{aligned}
\delta \Pi_{\mathrm{E}}^{e} & =\int_{\Omega_{\sigma}^{e}}\left[\delta \boldsymbol{u}_{0} \cdot \overline{\mathbf{N}}+\delta \boldsymbol{\theta} \cdot \overline{\mathbf{M}}+\delta \boldsymbol{\psi}_{1} \cdot \overline{\mathbf{M}}_{\phi_{1}}+\delta \boldsymbol{\psi}_{2} \cdot \overline{\mathbf{M}}_{\phi_{2}}\right] \mathrm{d} \Gamma^{e} \\
& +\int_{\Omega^{e}}\left(\left.\delta \boldsymbol{u}\right|_{\xi^{3}(0)} q^{(0)} \mu^{(0)}-\left.\delta \boldsymbol{u}\right|_{\xi^{3}(N)} q^{(N)} \mu^{(N)}\right) \cdot \boldsymbol{a}_{3} \mathrm{~d} \Omega^{e},
\end{aligned}
$$

where the stress resultants due to the prescribed traction acting on the Neumann boundary are defined as follows

$$
\begin{aligned}
\langle\overline{\mathbf{N}}, \overline{\mathbf{M}}\rangle & =\int_{h}\left\langle 1, \xi^{3}\right\rangle \overline{\boldsymbol{h}} \mu \mathrm{d} \xi^{3}, \\
\left\langle\overline{\mathbf{M}}_{\phi_{1}}, \overline{\mathbf{M}}_{\phi_{2}}\right\rangle & =\int_{h}\left\langle\phi_{1}^{(k)}, \phi_{2}^{(k)}\right\rangle \overline{\boldsymbol{h}} \mu \mathrm{d} \xi^{3} .
\end{aligned}
$$

\section{Computational formulation}

In this section the weak form derived in Section 5 is employed to develop a bilinear shell element. Assumed Natural Strain strategy and Enhanced Assumed Strain technique are applied to alleviate the shear locking introduced by the bilinear shape functions as well as to increase element's performance. Efficiency of the finite element is increased by using a one-point quadrature rule with hourglass stabilization for the in-plane integration. Moreover, the element's stiffness matrix is obtained from linearization of the residuals.

\subsection{Spatial interpolation and shear locking}

Introducing the $\mathrm{C} 0$-continuous interpolation of the kinematic variables into Eq. (24) allows the displacement field to be rewritten as

$$
\boldsymbol{u}^{(k)}=\sum_{I=1}^{4} \mathrm{~N}^{I}\left(\boldsymbol{u}_{0}^{I}+\xi^{3} \boldsymbol{\theta}^{I}+\phi_{1}^{(k)} \boldsymbol{\psi}_{1}^{I}+\phi_{2}^{(k)} \boldsymbol{\psi}_{2}^{I}\right)
$$

where $\mathrm{N}^{I}\left(\xi^{\alpha}\right)$ denote the standard isoparametric bilinear shape functions [48] which map a point on the reference surface from the reference bi-unit square, $[-1,1]^{2}$, to the physical space and the $I=1, \ldots, 4$ superscript is used to indicate quantities evaluated at the $I$-th node.

The reference surface's normal and curvature are necessary to compute the strain resultants in Eqs. (26)-(32). Therefore, the director field of a smooth surface is given as an averaged value [49] and the curvatures are obtained using the procedure described in [47].

When thin structures are studied, the element's performances are affected by transverse shear locking. The aforementioned mesh locking is alleviated through the Assumed Natural Strain (ANS) approach $[38,50]$ by applying the well known MITC4 interpolation scheme [39].

\subsection{Enhanced strain interpolation}

Efficiency of EAS method depends on the choice of the interpolation for the strain enhancements. Several works focused on the optimal choice of EAS interpolation to mitigate finite element parasitic phenomena, Refs. [40, 41, 47, 51] among others. In the present work membrane strain enrichments and bending strain enrichments, respectively $\hat{\boldsymbol{\varepsilon}}^{\mathrm{m}}$ and $\hat{\boldsymbol{\varepsilon}}^{\mathrm{b}}$, are introduced to increase element's efficiency in membrane-dominated and bending-dominated problems. It order to fulfill the patch test requirements, it is necessary to map the strain enrichment, $\tilde{\boldsymbol{\varepsilon}}$, to the center of the element [47], $\xi^{1}=\xi^{2}=0$, through the following mapping

$$
\tilde{\boldsymbol{\varepsilon}}=\frac{\mathbf{J}}{\hat{\mathrm{J}}} \hat{\varepsilon}_{i j} \hat{\boldsymbol{g}}^{i} \otimes \boldsymbol{g}^{j}
$$

where $\left.\mathbf{J}=\operatorname{det}\left(\left[\boldsymbol{g}_{1}, \boldsymbol{g}_{2}, \boldsymbol{g}_{3}\right]\right), \hat{\mathbf{J}}=\operatorname{det}\left(\left[\hat{\boldsymbol{g}}_{1}, \hat{\boldsymbol{g}}_{2}, \hat{\boldsymbol{g}}_{3}\right]\right)\right)$, where $\hat{\boldsymbol{g}}_{i}$ and $\hat{\boldsymbol{g}}^{i}$ are the base vectors evaluated at the element center and where the resulting strain enrichment is given by

$$
\hat{\boldsymbol{\varepsilon}}=\hat{\boldsymbol{\varepsilon}}^{\mathrm{m}}+\xi^{3} \hat{\boldsymbol{\varepsilon}}^{\mathrm{b}}
$$

The components of the membrane and bending enrichments are given by

$$
\left\{\begin{array}{l}
\hat{\varepsilon}_{11}^{\mathrm{m}} \\
\hat{\varepsilon}_{22}^{\mathrm{m}} \\
\hat{\varepsilon}_{12}^{\mathrm{m}}
\end{array}\right\}=\hat{\mathbf{N}}^{\mathrm{m}} \boldsymbol{\alpha}^{\mathrm{m}}, \quad\left\{\begin{array}{l}
\hat{\varepsilon}_{11}^{\mathrm{b}} \\
\hat{\varepsilon}_{22}^{\mathrm{b}} \\
\hat{\varepsilon}_{12}^{\mathrm{b}}
\end{array}\right\}=\hat{\mathbf{N}}^{\mathrm{b}} \boldsymbol{\alpha}^{\mathrm{b}},
$$

where, due to the symmetry of the strain tensor, $\hat{\varepsilon}_{21}^{\mathrm{m}}=\hat{\varepsilon}_{12}^{\mathrm{m}}$ and $\hat{\varepsilon}_{21}^{\mathrm{b}}=\hat{\varepsilon}_{12}^{\mathrm{b}}$ and where $\boldsymbol{\alpha}^{\mathrm{m}}$ and $\boldsymbol{\alpha}^{\mathrm{b}}$ represent respectively the membrane and bending strain enhancement unknowns. Finally, a formulation employing ten EAS parameters, five for the membrane enrichment and five for the bending enrichment, is chosen and the interpolation matrices, $\hat{\mathbf{N}}^{\mathrm{m}}$ and $\hat{\mathbf{N}}^{\mathrm{b}}$, are given as

$$
\hat{\mathbf{N}}^{\mathrm{b}}=\hat{\mathbf{N}}^{\mathrm{m}}=\left[\begin{array}{ccccc}
\xi^{1} & 0 & 0 & 0 & 0 \\
0 & \xi^{2} & 0 & 0 & 0 \\
0 & 0 & \xi^{1} & \xi^{2} & \xi^{1} \xi^{2}
\end{array}\right]
$$




\subsection{Reduced integration and hourglass stabilization}

Reduced in-plane integration schemes rely on the possibility to express the displacement field, the covariant basis vectors and, consequently, the strain tensor in polynomial form $[42,52]$. Denoting the Cartesian coordinates of the $I$-th node of the shell with $\boldsymbol{X}^{I}$ and substituting the isoparametric mapping $\boldsymbol{r}\left(\xi^{\alpha}\right)=\sum_{I=1}^{4} \mathrm{~N}^{I} \boldsymbol{X}^{I}$ into Eq. (2) and Eq. (10) allows the covariant base vectors to be rewritten as follows

$$
\boldsymbol{g}=\boldsymbol{g}^{0}+\xi^{1} \boldsymbol{g}^{1}+\xi^{2} \boldsymbol{g}^{2}+\xi^{1} \xi^{2} \boldsymbol{g}^{3}
$$

After substituting Eq. (53) into Eqs. (26)-(32) the strain tensor can be rewritten as a sum of an in-plane constant term, $\boldsymbol{\varepsilon}^{0}$, and a hourglass part, $\boldsymbol{\varepsilon}^{h g}$, that collects the $\xi^{1}, \xi^{2}$ and $\xi^{1} \xi^{2}$ monomials

$$
\boldsymbol{\varepsilon}=\boldsymbol{\varepsilon}^{0}+\boldsymbol{\varepsilon}^{h g}
$$

where

$$
\varepsilon^{0}=\bar{\varepsilon}^{0}+\tilde{\varepsilon}^{0}
$$

with

$$
\boldsymbol{\varepsilon}^{h g} \approx \xi^{1}\left(\bar{\varepsilon}^{1}+\tilde{\varepsilon}^{1}\right)+\xi^{2}\left(\bar{\varepsilon}^{2}+\tilde{\varepsilon}^{2}\right)+\xi^{1} \xi^{2}\left(\bar{\varepsilon}^{3}+\tilde{\varepsilon}^{3}\right),
$$

where higher order monomials have been neglected and where the $k$ superscript has been omitted for the sake of readability. It should to be observed that the decomposition into constant part and hourglass part has been applied to the compatible strain tensor as well as to the strain enhancements. The material tangent moduli, $\mathbb{C}$, is computed in the material reference frame spanned by the orthonormal base vectors $\boldsymbol{e}_{i}$, with $\boldsymbol{e}_{3} \equiv \boldsymbol{a}_{3}$. Therefore, the strain tensor computed in the local curvilinear reference frame, $\boldsymbol{\varepsilon}$, is transformed to the material reference frame, $\overline{\boldsymbol{\epsilon}}$, through the mapping

$$
\bar{\epsilon}_{i j}=\left(\boldsymbol{e}_{i} \cdot \boldsymbol{g}^{k}\right) \varepsilon_{k l}\left(\boldsymbol{g}^{l} \cdot \boldsymbol{e}_{j}\right) .
$$

and the stress tensor in the material reference frame, $\bar{\sigma}$, is obtained from Eq. (40). The mapping in Eq. (57) is evaluated at the element center and it is assumed to be constant on the $\xi^{\alpha}$ space. Therefore, the element distortion is not considered [53]. Due to the decomposition of the strain tensor in polynomial form, the integrals appearing in the variational formulation of Section 5 can be computed analytically, accordingly to

$$
\begin{aligned}
\int_{-1}^{1} \int_{-1}^{1}\left(\xi^{\alpha}\right)^{2} \mathrm{~d} \xi^{1} \mathrm{~d} \xi^{2} & =\frac{4}{3} \\
\int_{-1}^{1} \int_{-1}^{1}\left(\xi^{1} \xi^{2}\right)^{2} \mathrm{~d} \xi^{1} \mathrm{~d} \xi^{2} & =\frac{4}{9} .
\end{aligned}
$$

Through-thickness integration is be carried out numerically, with quadrature points aligned at the center of the element and spanning the through-thickness coordinate.

\subsection{Residuals vector and tangent matrix}

For ease of notation, the nodal degrees of freedom of the element are collected within the $\boldsymbol{d}$ vector

$$
d=\left[d^{1}, d^{2}, d^{3}, d^{4}\right]^{T}
$$

where

$$
\boldsymbol{d}^{I}=\left[u_{1}^{I}, u_{2}^{I}, u_{3}^{I}, \theta_{1}^{I}, \theta_{2}^{I}, \psi_{1}^{I}, \psi_{2}^{I}\right] .
$$

Similarly, the enhancement unknowns are collected within the $\alpha$ vector

$$
\boldsymbol{\alpha}=\left\{\begin{array}{l}
\boldsymbol{\alpha}^{\mathrm{m}} \\
\boldsymbol{\alpha}^{\mathrm{b}} \\
\boldsymbol{\alpha}^{\mathrm{n}}
\end{array}\right\}
$$

The element residuals corresponding to the displacement unknowns, $\overline{\boldsymbol{r}}$, and strain enrichment unknowns, $\tilde{\boldsymbol{r}}$, are obtained from the weak form in Section 5 and are defined as follows

$$
\overline{\boldsymbol{r}}:=\frac{\partial\left(\Pi_{\mathrm{I}}^{e}-\Pi_{\mathrm{E}}^{e}\right)}{\partial \delta \boldsymbol{d}}, \quad \tilde{\boldsymbol{r}}:=\frac{\partial \Pi_{\mathrm{I}}^{e}}{\partial \delta \boldsymbol{\alpha}} .
$$

where $\delta \boldsymbol{d}$ and $\delta \boldsymbol{\alpha}$ respectively denote the virtual variation of $\boldsymbol{d}$ and the virtual variation of $\alpha$.

Owing to the locality of the strain enrichments, static condensation on the strain enrichment unknowns is performed and the element level residual vector and tangent matrix are given by

$$
\begin{aligned}
\mathbf{K}^{e} & =\overline{\mathbf{K}}-\widehat{\mathbf{K}}(\widetilde{\mathbf{K}})^{-1} \widehat{\mathbf{K}}^{T}, \\
\boldsymbol{r}^{e} & =\overline{\boldsymbol{r}}-\widehat{\mathbf{K}}(\widetilde{\mathbf{K}})^{-1} \tilde{\boldsymbol{r}} .
\end{aligned}
$$

where the $\overline{\mathbf{K}}, \widehat{\mathbf{K}}$ and $\widetilde{\mathbf{K}}$ matrices are defined as follows

$$
\overline{\mathbf{K}}:=\frac{\partial \overline{\boldsymbol{r}}}{\partial \boldsymbol{d}}, \quad \widehat{\mathbf{K}}:=\frac{\partial \overline{\boldsymbol{r}}}{\partial \boldsymbol{\alpha}}, \quad \widetilde{\mathbf{K}}:=\frac{\partial \tilde{\boldsymbol{r}}}{\partial \boldsymbol{\alpha}},
$$

Finally, the residual vector and tangent matrix computed for each element are assembled following the standard procedure [54].

\subsection{Constitutive relations and zigzag functions}

As observed in Section 4, the transverse normal strain is identically zero and the transverse normal stress, $\sigma_{33}$, is assumed to be zero as well (plane stress condition [3]). Therefore, the generalized Hooke's law for the $k$-th orthotropic lamina in Eq. (40) is given in the material reference frame in terms of plane stress reduced stiffnesses as

$$
\left\{\begin{array}{l}
\bar{\sigma}_{11} \\
\bar{\sigma}_{22} \\
\bar{\sigma}_{12} \\
\bar{\sigma}_{23} \\
\bar{\sigma}_{13}
\end{array}\right\}^{(k)}=\left[\begin{array}{ccccc}
\mathrm{C}_{11} & \mathrm{C}_{12} & \mathrm{C}_{16} & 0 & 0 \\
\mathrm{C}_{12} & \mathrm{C}_{22} & \mathrm{C}_{26} & 0 & 0 \\
\mathrm{C}_{16} & \mathrm{C}_{26} & \mathrm{C}_{66} & 0 & 0 \\
0 & 0 & 0 & \mathrm{Q}_{22} & \mathrm{Q}_{12} \\
0 & 0 & 0 & \mathrm{Q}_{12} & \mathrm{Q}_{11}
\end{array}\right]^{(k)}\left\{\begin{array}{c}
\bar{\epsilon}_{11} \\
\bar{\epsilon}_{22} \\
2 \bar{\epsilon}_{12} \\
2 \bar{\epsilon}_{23} \\
2 \bar{\epsilon}_{13}
\end{array}\right\}^{(k)}
$$

where the expression for the transformed elastic stiffness coefficient can be found in Ref. [3] and where Voigt notation has been employed [40].

In the current work, the zigzag functions characteristic of the $k$-th layer, $\phi_{\alpha}^{(k)}$ for $\alpha=1,2$, are assumed to be linear within the $k$-th layer $[26,55]$. The conditions necessary to obtain the 


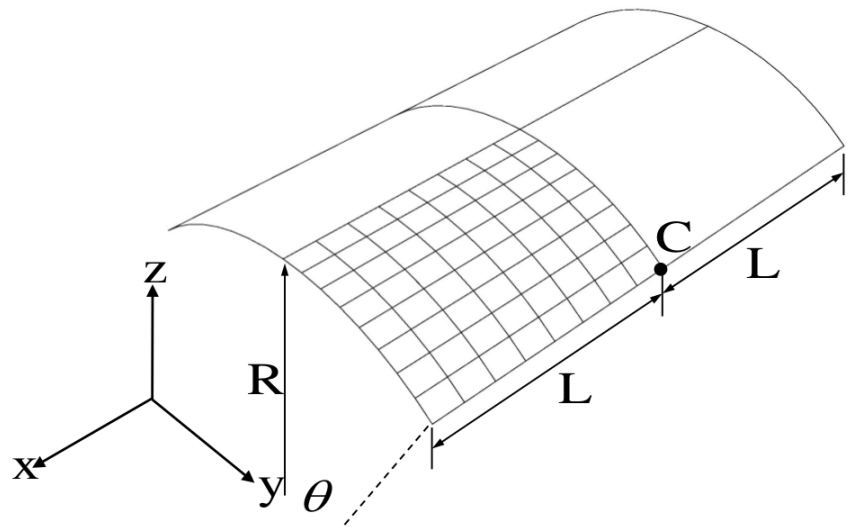

Figure 2: Scordelis-Lo roof: Geometry and FEM mesh.

coefficients of the zigzag functions are computed enforcing displacement continuity at each interface

$$
\left.\phi_{\alpha}^{(k)}\right|_{\xi^{3}=\xi^{3(k)}}=\left.\phi_{\alpha}^{(k+1)}\right|_{\xi^{3}=\xi^{3(k)}},
$$

for $k=1, \ldots, n-1$ and by constraining the zigzag displacement at the top and bottom surfaces

$$
\phi_{\alpha}^{(1)}(-h)=\phi_{\alpha}^{(N)}(h)=0 .
$$

Finally, the remaining conditions necessary to determine the zigzag functions are obtained from (Refs. [26, 55])

$$
\beta_{\alpha}^{(k)}=\frac{\mathrm{G}_{\alpha}}{\mathrm{Q}_{\alpha \alpha}^{(k)}}-1,
$$

with

$$
\mathrm{G}_{\alpha}=\left(\frac{1}{2 h} \sum_{k=1}^{n} \frac{2 h^{(k)}}{\mathrm{Q}_{\alpha \alpha}^{(k)}}-1\right)^{-1} .
$$

\section{Numerical examples}

In this section, numerical experiments are carried out to assess the performance of the element. First, two popular benchmark tests for shells are used to examine the convergence properties of the element in membrane-dominated and bendingdominated problems in the thin-shell regime [56, 57], Sec. 7.1 and 7.2. In order to fully exploit the zigzag kinematics, the homogeneous limit methodology is employed, Ref. [27], when monolithic laminates are considered. Next, the problem of a simply-supported laminated cylindrical shell is presented 7.3. The shell is submitted to bi-sinusoidal loading, several laminations are modeled and the results are compared with the known analytical solution due to Ren [58]. Accuracy of displacements as well as interfacial stresses is shown for thin and thick shells.

Material parameters, geometric dimensions, loads, displacement and stresses are given in consistent units of measure.

\subsection{Scordelis-Lo roof}

The Scordelis-Lo roof [56] is a membrane-dominated problem concerning the deformation of the short cylindrical shell, shown in Fig. 2, under its own weight (90 units of force per unit surface area, along the negative $\mathrm{z}$-direction). The structure is supported by rigid diaphragms $\left(u_{y} \equiv u_{z} \equiv 0\right)$ at the curved edges, while its straight edges are free. It has a length of $2 L=50$, thickness $2 h=0.25$, radius of curvature $R=25$, and $\theta=40^{\circ}$. The material is isotropic and homogeneous, with Young's modulus $E=4.32 \times 10^{8}$, Poisson's ratio $v=0$, and density $\rho=360$. Owing to symmetry, only one quadrant of the structure is modeled, using a mesh of $n_{e} \times n_{e}$ elements, as shown in Fig. 2. The laminate is discretized using ten computational layers and the homogeneous limit methodology in Ref. [27].

It should be remarked that the discretization of the homogeneous laminate into ten computational layers is only a numerical artifact that, together with the homogeneous limit methodology, gives non-zero zigzag functions through the thickness of the laminate. Therefore, this methodology allows to fully exploit RZT capabilities when homogeneous laminates are modeled.

Table 1: Scordelis-Lo roof: normalized vertical displacement at free edge mid-point (point $\mathrm{C}$ ).

\begin{tabular}{ccccc}
$n_{e}$ & Present element & Ref. [59] & Ref. [53] & Ref. [47] \\
\hline 2 & 1.396 & - & - & 1.198 \\
4 & 1.048 & 0.995 & 0.997 & 0.944 \\
8 & 1.006 & 0.986 & 0.994 & 0.973 \\
16 & 0.998 & 0.993 & 1.000 & 0.992 \\
32 & 0.996 & 0.996 & 1.003 & - \\
\hline
\end{tabular}

Table 1 gives the value of the vertical displacement at the mid-point of the roof's free edge (point C in Fig. 2), normalized with respect to the reference solution (0.3024), computed numerically by Scordelis and Lo [56]. Results obtained using the present formulation are compared, at different levels of mesh refinement, with those of the solid-shell elements of Alves De Souza et al. [59] and Schwarze and Reese [53], and the geometrically exact shell element of Simo et al. [47]. Clearly, as the mesh is refined, the results from the present element converge monotonically to the reference solution, at nearly the same rate as the elements of Refs. [53, 59] which employ EAS and reduced integration. It can be concluded that the present element does not suffer from membrane locking.

\subsection{Pinched hemi-spherical cap}

In this example, we consider a hemi-spherical shell with radius $R=10$, thickness $2 h=0.04$, and a hole cut out from the top as shown in Fig. 3 with $\theta=18^{\circ}$. The shell is made of an isotropic material with Young's modulus $E=6.825 \times 10^{7}$ and Poisson's ratio $v=0.3$. Making use of symmetry, we model one quarter of the cap only, using a mesh comprising $n_{e} \times n_{e}$ elements. Keeping its top and bottom edges traction-free, the shell is pinched by alternating radial point loads, acting along the $x$ and $y$-axes as shown in Fig. 3. As in the previous example, ten 
computational layers and homogeneous limit methodology are employed.

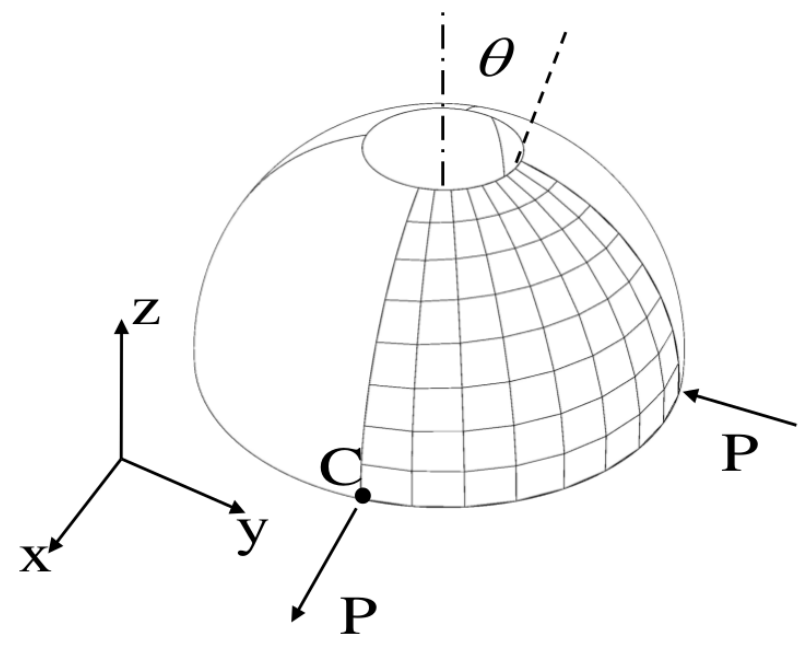

Figure 3: Hemi-spherical cap: Geometry and mesh $\left(n_{e}=8\right)$.

Table 2: Pinched hemi-spherical cap: normalized displacement at point of load application (point C).

\begin{tabular}{ccccc}
$n_{e}$ & Present element & Ref. [53] & Ref. [60] & Ref. [47] \\
\hline 4 & 0.984 & 1.043 & 1.032 & 0.993 \\
8 & 0.987 & 1.002 & 0.999 & 0.987 \\
16 & 0.990 & 0.993 & 0.993 & 0.988 \\
32 & 0.993 & 0.994 & 0.995 & - \\
\hline
\end{tabular}

The radial displacement, computed at the point of load application (point $\mathrm{C}$ in Fig. 3), is normalized with respect to the reference value of 0.094 , computed by MacNeal and Harder [57]. These results, presented in Table 2 for different mesh densities, are compared to those of the solid-shell elements of Refs. [53, 60] and the geometrically exact shell element of Simo et al. [47] (the values given in Refs. [47, 60] have been re-normalized to match the reference value of 0.094). Shell elements of Ref. [53, 60] and geometrically exact shell element of Ref. [47] converge non-monotonically respectively from above and from below whereas the present element converges monotonically from below. Nevertheless, the elements of Refs. [53, 60] show slightly better performance. Accordingly, it can be stated that the present formulation is free from shear locking.

\subsection{Simply-supported laminated cylindrical shell}

In this section, we consider the laminated cylindrical shell shown in Fig. 4, with dimensions $L=10, R=5$ and $\Phi=\pi / 2$. The shell is subject to bi-sinusoidal loads, applied to its top and bottom surfaces, of amplitude $q^{(0)}=q^{(N)}=5 \times 10^{-3}$. Each edge is subdivided into $n_{e}$ equal segments, defining an $n_{e} \times n_{e}$ mesh. The material mechanical properties are shown in Table 3 and the three laminates considered in the present section are listed in Tab. 4 where the 0 angle coincides with the axial direction of the cylindrical shell. Relative error on the maximum deflection is computed for several radius to thickness ratios, $S=R /(2 h)$, and the through-thickness distribution of stresses is shown.

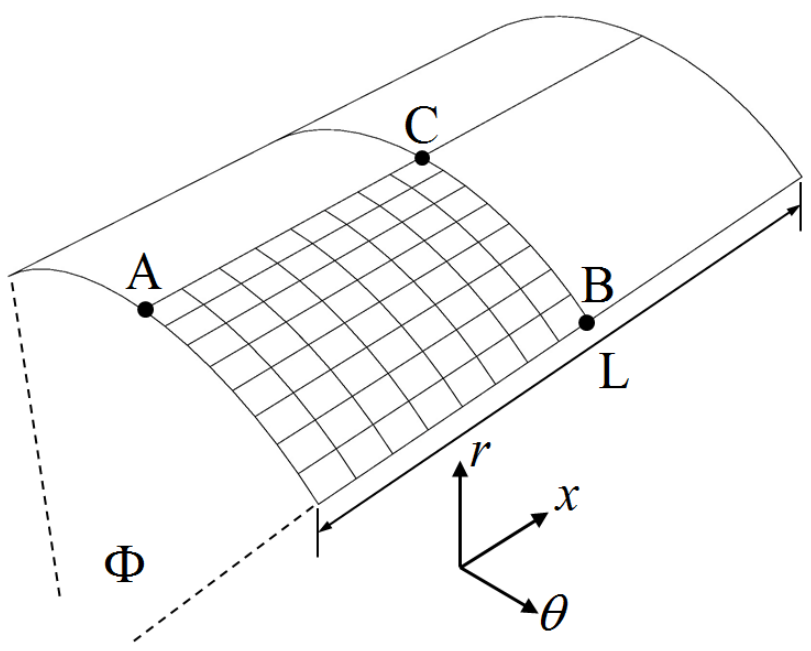

Figure 4: Laminated cylindrical shell: Geometry and FEM $\operatorname{mesh}\left(n_{e}=8\right)$.

In the current section the following notation is introduced to identify the formulations obtained modifying the displacement field in Eq. (48): 1) FSDT denotes the finite element that doesn't employ the zigzag kinematics, $\phi_{\alpha} \equiv 0$ for $\alpha=1,2$ and uses shear correction factors equal to $5 / 6,2$ ) RZT denotes the shell finite element that employs the full zigzag kinematics without shear correction factors. The reference radial displacement, $u_{r}^{r e f}$, computed at C, Fig. 4, is obtained from the integral average of the analytic $u_{r}$ distribution computed from Ren's solution [58]

$$
u_{r}^{r e f}=\frac{1}{2 h} \int_{h} u_{r}\left(\xi^{3}, \Phi / 2, L / 2\right) \mathrm{d} \xi^{3} .
$$

The values of the reference deflection for the considered laminations and radius to thickness ratios are given in Tab. 5. The relative error of the solution computed with the FSDT and RZT finite element formulations is indicated with $e_{r}=1-u_{r} / u_{r}^{r e f}$.

Table 3: Material mechanical properties.

\begin{tabular}{lccccc} 
& $\mathrm{A}$ & $\mathrm{B}$ & $\mathrm{C}$ & $\mathrm{D}$ & $\mathrm{E}$ \\
\hline$E_{1}$ & 50.0 & 157.9 & 250.0 & & \\
$E_{2}$ & 10.0 & 9.6 & 10.0 & $1.0 \times 10^{-3}$ & 0.10 \\
$E_{3}$ & 10.0 & 9.6 & 10.0 & & \\
$v_{12}$ & 0.25 & 0.32 & 0.25 & & \\
$v_{13}$ & 0.25 & 0.32 & 0.25 & 0.01 & 0.33 \\
$v_{23}$ & 0.25 & 0.49 & 0.25 & & \\
$G_{12}$ & 5.0 & 5.9 & 5.0 & & \\
$G_{13}$ & 5.0 & 5.9 & 5.0 & & \\
$G_{23}$ & 5.0 & 3.2 & 2.0 & & \\
\hline
\end{tabular}

In Tabs. 6-8 the percentage relative error on the radial displacement, $e_{r}$, is presented for the three considered laminations. For each laminate, the relative error obtained 
Table 4: Stacking information for laminates I-III.

\begin{tabular}{cccc} 
& Stacking sequence* & Orientation & Relative thickness $\left(h^{(k)} / h\right)$ \\
\hline I & A/A/D/A/A & {$[0 / 90 / 0 / 90 / 0]$} & {$[0.05,0.05,0.80,0.05,0.05]$} \\
II & B/E/B & {$[0 / 0 / 0]$} & {$[0.10,0.80,0.10]$} \\
III & C/C/C & {$[0 / 90 / 0]$} & {$[1 / 3,1 / 3,1 / 3]$} \\
\hline${ }^{*}$ Properties of materials A-E are given in Table 3. & \\
\hline
\end{tabular}

Table 5: Reference deflection at C, $u_{r}^{r e f}$.

\begin{tabular}{cccc}
\hline $\mathrm{S}$ & Laminate I & Laminate II & Laminate III \\
\hline 4 & 0.4268 & 0.3662 & 0.0274 \\
6 & 0.6457 & 0.5811 & 0.0519 \\
10 & 1.081 & 1.038 & 0.116 \\
20 & 2.165 & 2.271 & 0.307 \\
100 & 10.856 & 13.030 & 1.781 \\
\hline
\end{tabular}

with the RZT-based shell element is shown for several mesh densities $\left(n_{e}=2,4,8,16,32\right)$ and radius to thickness ratios $(S=4,6,10,20,100)$. Moreover, results obtained from the FSDT formulation results are only shown for the converged value obtained with the finest mesh, $n_{e}=32$. It can clearly be stated that the RZT solution is convergent for all the considered laminations and radius to thickness ratios. Despite the fact that the RZT converged solution is not always coincident with the analytical reference solution, the RZT relative error is in general small and RZT performs better than FSDT. It is worth noticing that the magnitude of the relative error, $e_{r}$, depends on the shell's thickness and lamination and that when thin, $S \geq 20$, laminated shells are considered, both RZT ans FSDT solutions converge to the analytical value although RZT is more accurate than FSDT.

When thick sandwich-like laminates are considered (laminates I and II), FSDT significantly underestimates the maximum deflection, up to over $76 \%$, whereas the RZT predictions are excellent for the entire range of $S$ ratios considered, see Tabs. 6-7. The superior modeling capabilities of RZT are also demonstrated in Fig. 5 where the zigzag through-thickness distribution of the in-plane tangential displacement, $u_{\theta}$, is considered. As the mesh is refined, the RZT through-thickness distribution converges to the analytical solution whereas FSDT is unable to reproduce this behavior. Therefore, RZT allows to describe accurately the through-thickness distribution of in-plane normal stresses, $\sigma_{\theta \theta}$ and $\sigma_{x x}$, as demonstrated in Figs. 6 and 7. Conversely, application of FSDT leads to predict throughthickness distributions that are highly inaccurate. Figures 8 and 9 show that a piecewise constant through-the-thickness distribution of the transverse shear stresses is obtained, using Hooke's law, from both FSDT and RZT. The magnitude of the transverse shear stress computed from FSDT is overestimated in the stiffer layers (i.e. faces) and underestimated in the more compliant layers (i.e. core) whereas RZT provides a reasonable approximation of the average of the true stress distribution in each layer. Therefore, reliable values of the transverse shear stresses at $\xi^{3}= \pm h$ cannot be inferred when
Hooke's law is used to compute these stresses, i.e., the stresses will not match exactly the prescribed transverse shear tractions along these boundaries. On the other hand, integration of the equilibrium equations of three-dimensional elasticity theory, involving derivatives of the in-plane stresses, generally yields highly accurate results for the transverse shear stresses across the laminate thickness, including those at the bounding surfaces $\xi^{3}= \pm h$. This is because the through-thickness distributions of the in-plane stresses obtained with RZT are highly accurate (e.g., refer to [24, 31, 32, 55]).

From Tab. 8 it is evident that when thick composite laminates (laminates III) are modeled, RZT performs better than FSDT. Nevertheless, the relative error on the radial displacement at $\mathrm{C}$ is higher than the corresponding value obtained for sandwichlike laminates (laminate I and II). The reason for that behavior is related to the poor prediction of the through-thickness distribution of shear stresses, Figs. 10 and 11. As evinced from Figs. 12 and 13 application of RZT produces accurate description of in-plane normal stresses. Therefore, it may be possible to obtain better results by menas of a mixed formulation of RZT as detailed in [36].

Table 6: Laminate I: Relative error on deflection.

\begin{tabular}{cccccc}
\hline RZT & \multicolumn{5}{c}{$e_{r}=1-u_{r} / u_{r}^{r e f},(\%)$} \\
\cline { 2 - 6 }$n_{e}$ & $\mathrm{~S}=4$ & $\mathrm{~S}=6$ & $\mathrm{~S}=10$ & $\mathrm{~S}=20$ & $\mathrm{~S}=100$ \\
\hline 2 & 12.380 & 12.767 & 12.954 & 13.028 & 13.073 \\
4 & 2.613 & 3.043 & 3.251 & 3.331 & 3.353 \\
8 & 0.099 & 0.539 & 0.752 & 0.833 & 0.848 \\
16 & -0.535 & -0.092 & 0.122 & 0.204 & 0.217 \\
32 & -0.693 & -0.250 & -0.035 & 0.046 & 0.059 \\
\hline \hline \multirow{2}{*}{ FSDT } & \multicolumn{5}{c}{$e_{r}=1-u_{r} / u_{r}^{r e f},(\%)$} \\
$n_{e}$ & $\mathrm{~S}=4$ & $\mathrm{~S}=6$ & $\mathrm{~S}=10$ & $\mathrm{~S}=20$ & $\mathrm{~S}=100$ \\
\hline 32 & 72.583 & 57.832 & 34.936 & 12.017 & 0.361 \\
\hline
\end{tabular}

\section{Conclusions}

A generalization of the Refined Zigzag Theory (RZT) to doubly-curved multilayer composite and sandwich structures has been derived. The proposed formulation has been employed to develop a four node shell element with reduced integration and hourglass stabilization. A one-point quadrature rule is employed for the in-plane integration and numerical integration is carried out in the through-thickness coordinate whereas integration of the hourglass contribution is performed analytically. ANS strategy has been applied to overcome the shear locking 
Table 7: Laminate II: Relative error on deflection.

\begin{tabular}{cccccc}
\hline RZT & \multicolumn{5}{c}{$e_{r}=1-u_{r} / u_{r}^{r e f},(\%)$} \\
\cline { 2 - 6 }$n_{e}$ & $\mathrm{~S}=4$ & $\mathrm{~S}=6$ & $\mathrm{~S}=10$ & $\mathrm{~S}=20$ & $\mathrm{~S}=100$ \\
\hline 2 & 11.773 & 12.277 & 13.033 & 13.718 & 13.732 \\
4 & 4.088 & 4.324 & 4.641 & 4.595 & 3.621 \\
8 & 2.188 & 2.346 & 2.533 & 2.273 & 1.017 \\
16 & 1.715 & 1.852 & 2.006 & 1.690 & 0.361 \\
32 & 1.597 & 1.729 & 1.874 & 1.544 & 0.197 \\
\hline \hline \multirow{5}{*}{$\mathrm{FSDT}$} & \multicolumn{5}{c}{$e_{r}=1-u_{r} / u_{r}^{r e f},(\%)$} \\
$n_{e}$ & $\mathrm{~S}=4$ & $\mathrm{~S}=6$ & $\mathrm{~S}=10$ & $\mathrm{~S}=20$ & $\mathrm{~S}=100$ \\
\hline 32 & 76.835 & 68.197 & 49.513 & 18.812 & 0.302 \\
\hline
\end{tabular}

Table 8: Laminate III: Relative error on deflection.

\begin{tabular}{cccccc}
\hline RZT & \multicolumn{5}{c}{$e_{r}=1-u_{r} / u_{r}^{r e f},(\%)$} \\
\cline { 2 - 6 }$n_{e}$ & $\mathrm{~S}=4$ & $\mathrm{~S}=6$ & $\mathrm{~S}=10$ & $\mathrm{~S}=20$ & $\mathrm{~S}=100$ \\
\hline 2 & 12.286 & 12.379 & 12.373 & 12.318 & 12.286 \\
4 & 6.546 & 5.491 & 4.167 & 3.351 & 3.149 \\
8 & 5.136 & 3.767 & 2.082 & 1.052 & 0.798 \\
16 & 4.785 & 3.336 & 1.559 & 0.474 & 0.205 \\
32 & 4.697 & 3.229 & 1.428 & 0.329 & 0.057 \\
\hline \hline \multirow{2}{*}{ FSDT } & \multicolumn{5}{c}{$e_{r}=1-u_{r} / u_{r}^{r e f},(\%)$} \\
$n_{e}$ & $\mathrm{~S}=4$ & $\mathrm{~S}=6$ & $\mathrm{~S}=10$ & $\mathrm{~S}=20$ & $\mathrm{~S}=100$ \\
\hline 32 & 18.150 & 12.154 & 4.733 & 0.739 & 0.058 \\
\hline \multicolumn{5}{c}{}
\end{tabular}

due to the bilinear in-plane interpolation whereas EAS technique has been employed to improve the shell element's performance in membrane-dominated and bending-dominated problems.

Efficiency and accuracy of the present element have been assessed by way of numerical experiments. It has been shown that, when homogeneous curved structures are modeled, the element's performances and convergence rate are comparable to those of highly optimized shell elements. Nonetheless, the proposed formulation has been shown to provide great accuracy when highly heterogeneous composite laminates are considered in thin as well as thick regime. Substantial improvements over the widely used FSDT based finite elements have been obtained in the computation of the through-thickness distributions of displacements and stresses.

\section{Acknowledgments}

The first author gratefully acknowledges the support of this work by the Joint DoD/DOE Munitions Technology Development Program (JMP).

\section{References}

[1] D. Liu, X. Li, An overall view of laminate theories based on displacement hypothesis, J. Compos. Mater. 30 (1996) 1539-1561.

[2] E. Reissner, On a certain mixed variational theorem and a proposed application, Int. J. Numer. Meth. Engrg. 20 (1984) 1366-1368.

[3] J. N. Reddy, Mechanics of laminated composite plates, CRC Press, New York, 1997.

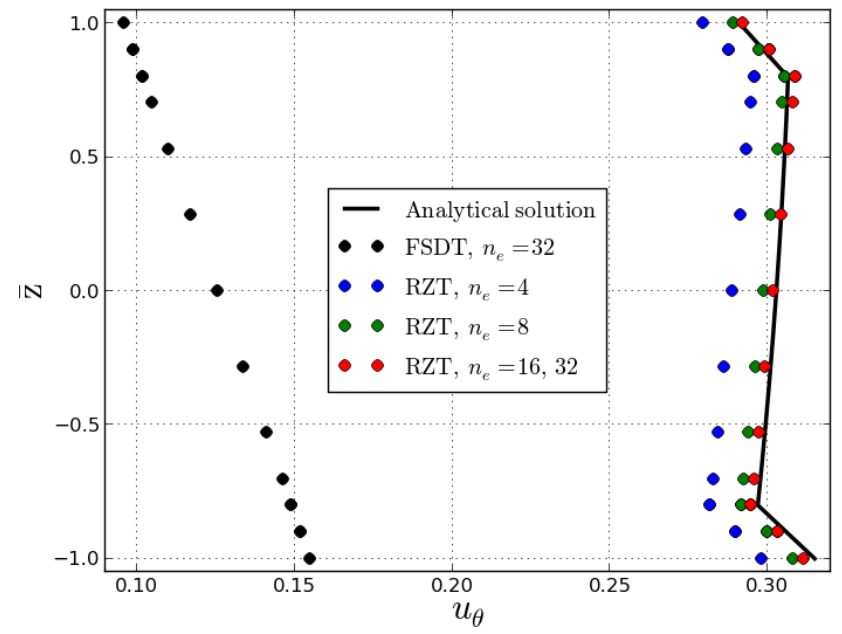

Figure 5: Laminate I, $S=6$, through-thickness $u_{\theta}$ distribution at point $\mathrm{B}$.

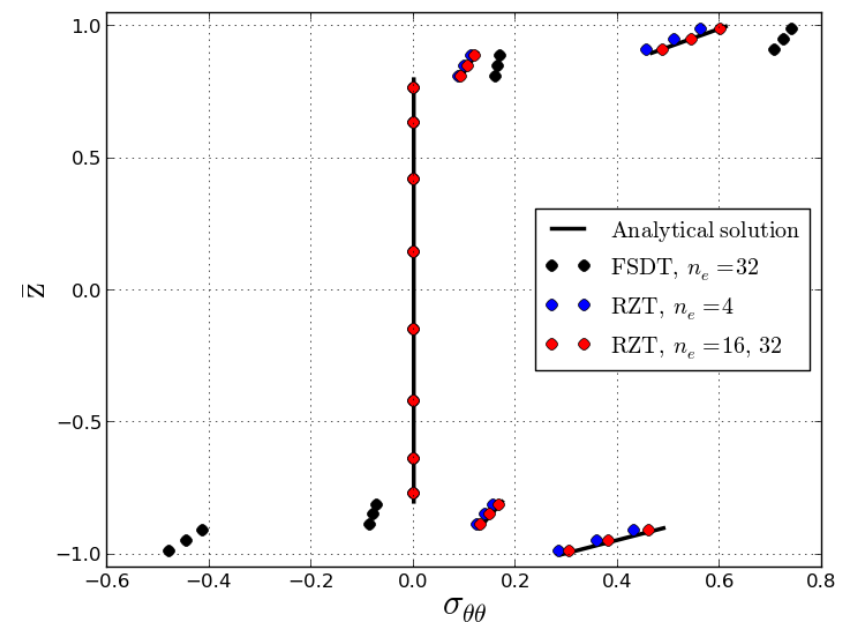

Figure 6: Laminate I, $S=6$, through-thickness $\sigma_{\theta \theta}$ stress distribution at point $\mathrm{C}$.

[4] E. Reissner, Y. Stavsky, Bending and stretching of certain types of heterogeneous aeolotropic elastic plates, J. Appl. Mech. 28 (1961) 402-408.

[5] E. Reissner, The effect of transverse shear deformation on the bending of elastic plates., J. Appl. Mech. 12 (1945) 68-77.

[6] R. Mindlin, Influence of rotatory inertia and shear deformation on flexural motions of isotropic elastic plates, J. Appl. Mech. 18 (1951) 31-38.

[7] J. Reddy, A simple higher-order theory for laminated composite plates, J. Appl. Mech. 51 (1984) 745-752.

[8] G. M. Cook, A. Tessler, A 3,2-order bending theory for laminated composite and sandwich beams, Composites Part B. 29 (1998) 565-576.

[9] M. Touratier, An efficient standard plate theory, Int. J. Eng. Sci. 29 (1991) 901-916.

[10] J. N. Reddy, A generalization of two-dimensional theories of laminated composite plates, Commun. Appl. Numer. Meth. 3 (1987) 173-180.

[11] X. Lu, D. Liu, An interlaminar shear stress continuity theory for both thin and thick composite laminates, J. Appl. Mech. 59 (1992) 502-509.

[12] M. Di Sciuva, A refinement of the transverse shear deformation theory for multilayered orthotropic plates, in: Proceedings of the 7th AIDAA National Congress.

[13] M. Di Sciuva, Development of an anisotropic, multilayered, sheardeformable rectangular plate element, Comput. Struct. 21 (1985) 789796.

[14] M. Di Sciuva, Multilayered anisotropic plate models with continuous 


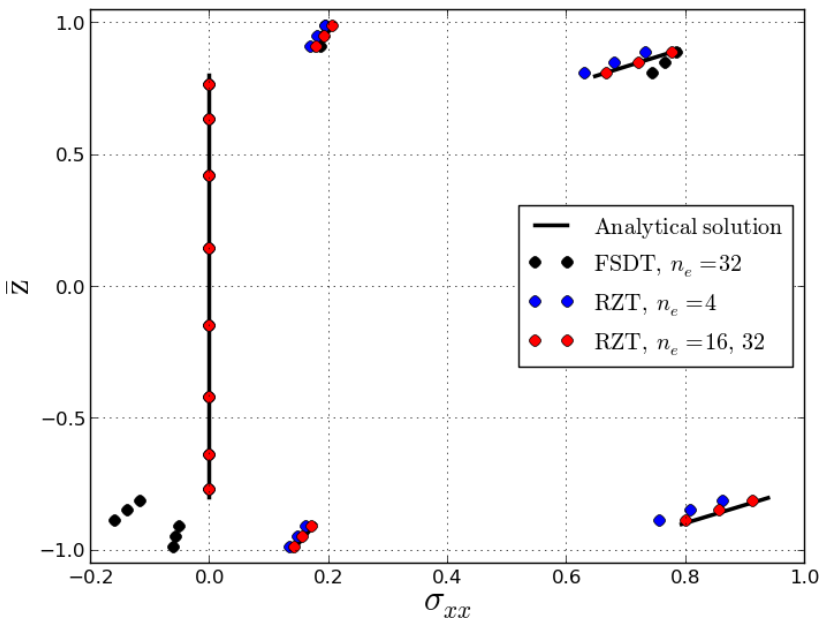

Figure 7: Laminate I, $S=6$, through-thickness $\sigma_{x x}$ stress distribution at point $\mathrm{C}$.

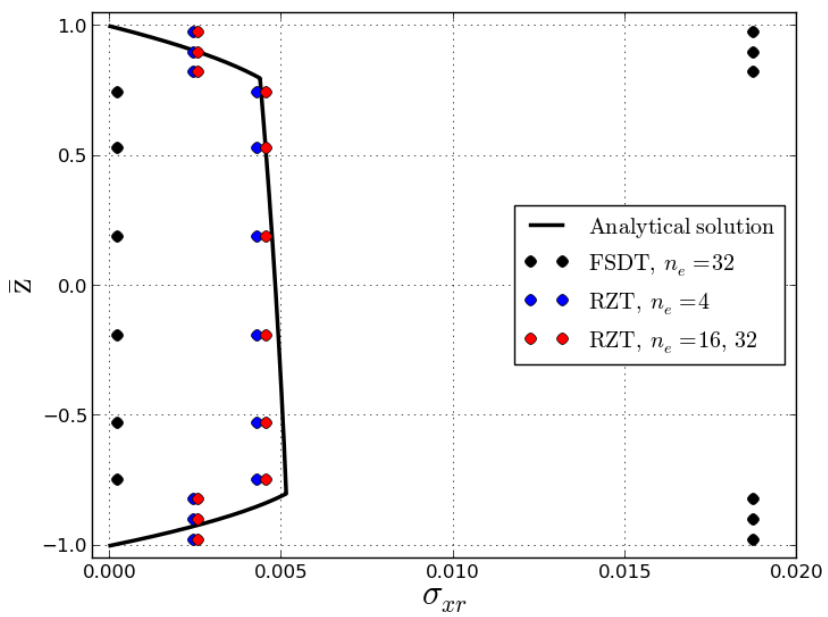

Figure 8: Laminate II, $S=6$, through-thickness $\sigma_{x r}$ stress distribution at point $\mathrm{A}$.

interlaminar stresses, Compos. Struct. 22 (1992) 149-167.

[15] M. Cho, R. Parmerter, Efficient higher order composite plate theory for general lamination configurations, AIAA Journal 31 (1993) 1299-1306.

[16] R. C. Averill, Static and dynamic response of moderately thick laminated beams with damage, Compos. Engrg. 4 (1994) 381-395.

[17] R. Averill, Y. C. Yip, Development of simple, robust finite elements based on refined theories for thick laminated beams, Comput. Struct. 59 (1996) $529-546$.

[18] U. Icardi, Higher-order zig-zag model for analysis of thick composite beams with inclusion of transverse normal stress and sublaminates approximations, Composites Part B. 32 (2001) 343-354.

[19] H. Arya, A new zig-zag model for laminated composite beams: free vibration analysis, J. Sound Vib. 264 (2003) 485-490.

[20] S. Kapuria, S. Kulkarni, An efficient quadrilateral element based on improved zigzag theory for dynamic analysis of hybrid plates with electroded piezoelectric actuators and sensors, J. Sound Vib. 315 (2008) 118 145.

[21] P. Vidal, O. Polit, A family of sinus finite elements for the analysis of rectangular laminated beams, Compos. Struct. 84 (2008) 56-72.

[22] G. Akhras, W. Li, Stability and free vibration analysis of thick piezoelectric composite plates using spline finite strip method, Int. J. Mech. Sci. 53 (2011) 575-584.

[23] R. Xiaohui, C. Wanji, W. Zhen, A c0-type zigzag theory and finite element for laminated composite and sandwich plates with general configu-

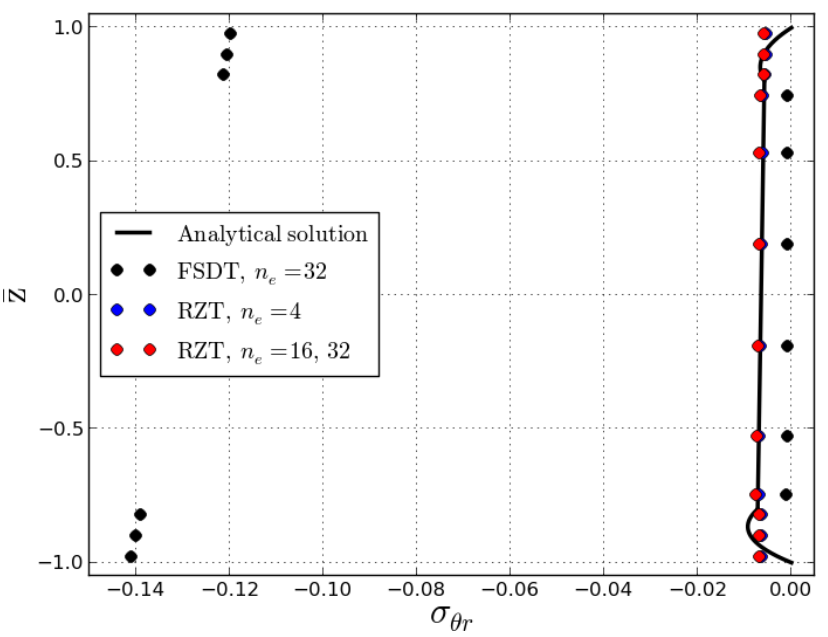

Figure 9: Laminate II, $S=6$, through-thickness $\sigma_{\theta r}$ stress distribution at point $\mathrm{B}$.

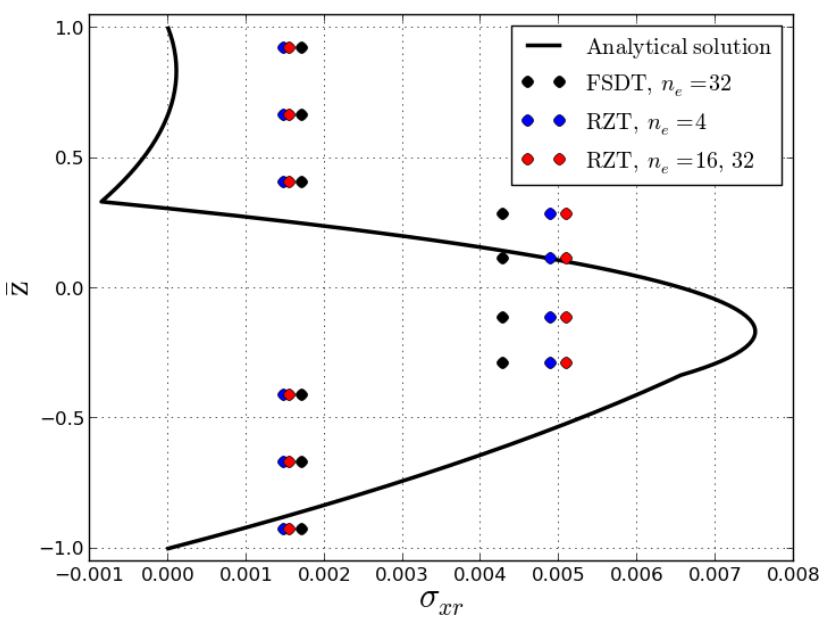

Figure 10: Laminate III, $S=6$, through-thickness $\sigma_{x r}$ stress distribution at point $\mathrm{A}$.

rations, Arch. Appl. Mech. 82 (2012) 391-406.

[24] A. Tessler, M. Di Sciuva, M. Gherlone, A refined zigzag beam theory for composite and sandwich beams, J. Compos. Mater. 43 (2009) 1051-1081.

[25] M. Di Sciuva, M. Gherlone, A. Tessler, A robust and consistent firstorder zigzag theory for multilayered beams, in: R. Gilat, L. Banks-Sills (Eds.), Advances in Mathematical Modeling and Experimental Methods for Materials and Structures, volume 168, 2010, pp. 255-268.

[26] A. Tessler, M. Di Sciuva, M. Gherlone, A consistent refinement of firstorder shear-deformation theory for laminated composite and sandwich plates using improved zigzag kinematics, J. Mech. Mater. and Struct. 5 (2010) 341-367.

[27] A. Tessler, M. Di Sciuva, M. Gherlone, A homogeneous limit methodology and refinements of computationally efficient zigzag theory for homogeneous, laminated composite, and sandwich plates, Numerical Methods for Partial Differential Equations 27 (2011) 208-229.

[28] L. Iurlaro, M. Gherlone, M. Di Sciuva, A. Tessler, Assessment of the refined zigzag theory for bending, vibration, and buckling of sandwich plates: a comparative study of different theories, Compos. Struct. 106 (2013) 777-792.

[29] M. Gherlone, A. Tessler, M. Di Sciuva, C0 beam elements based on the refined zigzag theory for multilayered composite and sandwich laminates, Compos. Struct. 93 (2011) 2882-2894.

[30] E. Oñate, A. Eijo, S. Oller, Simple and accurate two-noded beam element for composite laminated beams using a refined zigzag theory, Comput. 


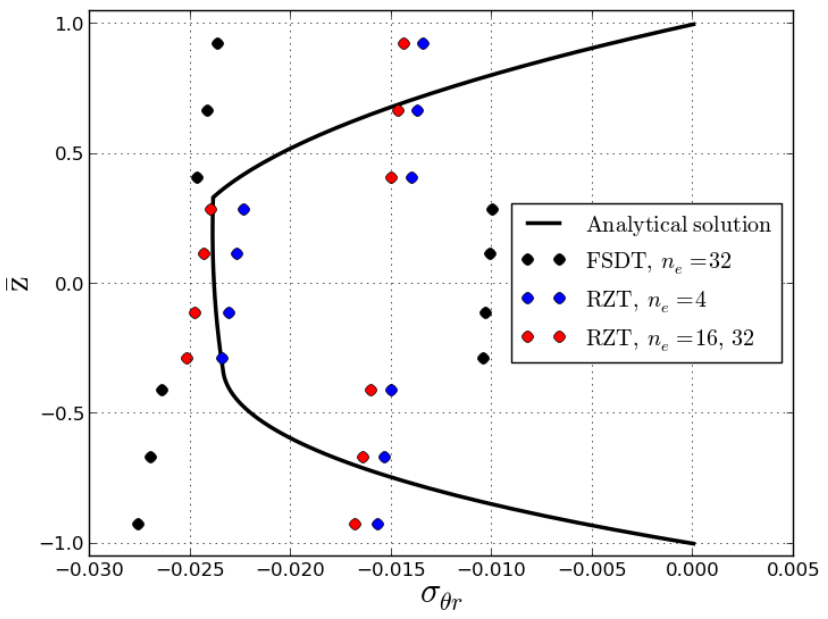

Figure 11: Laminate III, $S=6$, through-thickness $\sigma_{\theta r}$ stress distribution at point $\mathrm{B}$.

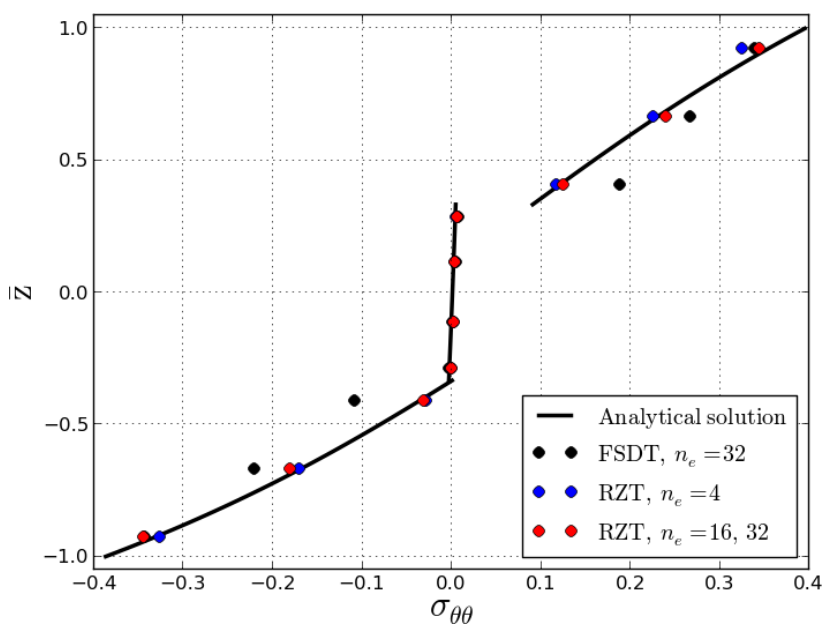

Figure 12: Laminate III, $S=6$, through-thickness $\sigma_{\theta \theta}$ stress distribution at point $\mathrm{C}$.

Methods Appl. Mech. Engrg. 213-216 (2012) 362-382.

[31] D. Versino, M. Gherlone, M. Mattone, M. Di Sciuva, A. Tessler, C0 triangular elements based on the refined zigzag theory for multilayer composite and sandwich plates, Composites Part B. 44 (2013) 218-230.

[32] A. Eijo, E. Oñate, S. Oller, A four-noded quadrilateral element for composite laminated plates/shells using the refined zigzag theory, Int. J. Numer. Meth. Engrg. 95 (2013) 631-660.

[33] A. Eijo, E. Oñate, S. Oller, A numerical model of delamination in composite laminated beams using the LRZ beam element based on the refined zigzag theory, Compos. Struct. 104 (2013) 270-280.

[34] A. Eijo, E. Oñate, S. Oller, Delamination in laminated plates using the 4noded quadrilateral qlrz plate element based on the refined zigzag theory, Compos. Struct. 108 (2014) 456-471.

[35] M. Gherlone, On the use of zigzag functions in equivalent single layer theories for laminated composite and sandwich beams: a comparative study and some observations on external weak layers, J. Appl. Mech. 80 (2013).

[36] L. Iurlaro, M. Gherlone, M. Di Sciuva, A. Tessler, A multi-scale refined zigzag theory for multilayered composite and sandwich plates with improved transverse shear stresses, in: Porceedings of the V International Conference on Coupled Problems in Science and Engineering.

[37] A. Barut, E. Madenci, A. Tessler, C0-continuous triangular plate element for laminated composite and sandwich plates using the 2,2 refined zigzag theory, Compos. Struct. 106 (2013) 835-853.

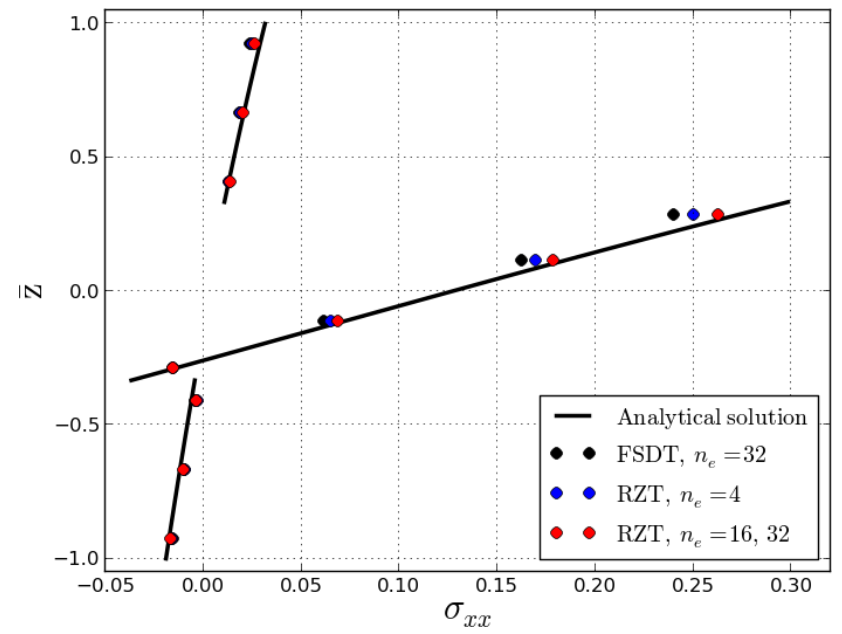

Figure 13: Laminate III, $S=6$, through-thickness $\sigma_{x x}$ stress distribution at point $\mathrm{C}$.

[38] T. J. R. Hughes, T. E. Tezduyar, Finite elements based upon Mindlin plate theory with particular reference to the four-node bilinear isoparametric element, J. Appl. Mech. 48 (1981) 587-596.

[39] E. N. Dvorkin, K.-J. Bathe, A continuum mechanics based four-node shell element for general non-linear analysis, Engrg. Comput. 1 (1984) $77-88$.

[40] L. Vu-Quoc, X. G. Tan, Optimal solid shells for non-linear analyses of multilayer composites. I. Statics, Comput. Methods Appl. Mech. Engrg. 192 (2003) 975-1016.

[41] M. Bischoff, W. A. Wall, K.-U. Bletzinger, E. Ramm, Models and finite elements for thin-walled structures, in: E. Stein, R. de Borst, T. J. R. Hughes (Eds.), Encyclopedia of Computational Mechanics, volume 2, Wiley, 2004, pp. 59-137.

[42] T. Belytschko, I. Leviathan, Physical stabilization of the 4-node shell element with one point quadrature, Comput. Methods Appl. Mech. Engrg. 113 (1994) 321-350.

[43] R. P. Cardoso, J. W. Yoon, One point quadrature shell element with through-thickness stretch, Comput. Methods Appl. Mech. Engrg. 194 (2005) 1161-1199.

[44] D. Chapelle, K. J. Bathe, Fundamental considerations for the finite element analysis of shell structures, Comput. Struct. 66 (1998) 19-36.

[45] R. A. Arciniega, J. N. Reddy, Tensor-based finite element formulation for geometrically nonlinear analysis of shell structures, Comput. Methods Appl. Mech. Engrg. 196 (2007) 1048-1073.

[46] A. E. Green, W. Zerna, Theoretical Elasticity, Oxford University Press, London, 2nd edition, 1968.

[47] J. C. Simo, D. D. Fox, M. S. Rifai, On a stress resultant geometrically exact shell model. Part II: The linear theory; computational aspects, Comput. Methods Appl. Mech. Engrg. 73 (1989) 53-92.

[48] O. C. Zienkiewicz, R. L. Taylor, The finite element method, ButterworthHeinemann, Oxford, 5th edition, 2000.

[49] Z. X. Li, B. A. Izzuddin, L. Vu-Quoc, A 9-node co-rotational quadrilateral shell element, Comput. Mech. 42 (2008) 873-884.

[50] R. H. Macneal, Derivation of element stiffness matrices by assumed strain distributions, Nucl. Engrg. Des. 70 (1982) 3-12.

[51] R. A. S. Moreira, R. J. Alves de Sousa, R. A. F. Valente, A solid-shell layerwise finite element for non-linear geometric and material analysis, Compos. Struct. 92 (2010) 1517-1523.

[52] T. Belytschko, C.-S. Tsay, A stabilization procedure for the quadrilateral plate element with one-point quadrature, Int. J. Numer. Meth. Engrg. 19 (1983) 405-419.

[53] M. Schwarze, S. Reese, A reduced integration solid-shell finite element based on the EAS and the ANS concept-Geometrically linear problems, Int. J. Numer. Meth. Engrg. 80 (2009) 1322-1355.

[54] T. J. R. Hughes, The finite element method: linear static and dynamic finite element analysis, Dover Publications, 2012.

[55] A. Tessler, M. Di Sciuva, M. Gherlone, Refined Zigzag Theory for Lam- 
inated Composite and Sandwich Plates, Technical Report NASA/TP2009-215561, NASA, 2009.

[56] A. C. Scordelis, K. S. Lo, Computer analysis of cylindrical shells, Amer. Concr. Inst. J. Proc. 61 (1964) 539-561.

[57] R. H. Macneal, R. L. Harder, A proposed standard set of problems to test finite element accuracy, Finite Elem. Anal. Des. 1 (1985) 3-20.

[58] J. G. Ren, Analysis of simply-supported laminated circular cylindrical shell roofs, Compos. Struct. 11 (1989) 277-292.

[59] R. J. Alves de Sousa, R. P. R. Cardoso, R. A. Fontes Valente, J.-W. Yoon, J. J. Grácio, R. M. Natal Jorge, A new one-point quadrature enhanced assumed strain (EAS) solid-shell element with multiple integration points along thickness: Part I-geometrically linear applications, Int. J. Numer. Meth. Engrg. 62 (2005) 952-977.

[60] F. Gruttmann, W. Wagner, A linear quadrilateral shell element with fast stiffness computation, Comput. Methods Appl. Mech. Engrg. 194 (2005) $4279-4300$. 